\title{
THE INTEGRABILITY PROBLEM FOR G-STRUCTURES( $\left.{ }^{(}\right)$
}

\author{
BY \\ VICTOR GUILLEMIN
}

Introduction. One of the main problems of local differential geometry is to determine when a given differentiable structure (for example, a Riemannian structure, a projective structure or an almost complex structure) is integrable. There are really two problems involved in this: (1) the problem of finding a consistent system of differential equations whose unknowns yield a solution to the problem; and (2) the problem of solving these equations. It goes without saying that the second problem is more or less trivial if we are considering analytic structures. On the other hand for $C^{\infty}$-structures it is extremely difficult, since the equations that occur in (1) are generally not elliptic.

In the paper we will only deal with the first problem which we will refer to as the problem of formal flatness or formal integrability. Also, for simplicity, we will restrict ourselves to studying $G$-structures, though most of the results of this paper can be extended to more general kinds of pseudogroup structures (such as projective structures).

We will also formulate the problem in slightly more intuitive terms: In $\$ 2$ we will define what we mean by a mapping of one $G$-structure into another which is structure preserving to kth order at some point, a notion which is almost self-evident.

We will try to find conditions on a given $G$-structure, $E \rightarrow M$, such that every point of $M$ admits a mapping into a neighborhood of an integrable $G$-structure which is structure preserving to order $k$ at that point, for arbitrarily large $k$. The obstructions to constructing such mappings turn out to be tensors of type $H^{k, 2}(g)$, defined on $E$, where the $H^{k, i}(g)$ are the bigraded homology groups of a certain chain complex due to Spencer (cf. \$1). Therefore, the character of the integrability problem will be determined by the cohomology sequence $H^{k, 2}(g)$.

We will also show in this paper that for $G$-structures of finite type formal integrability in the sense above implies integrability. For analytic $G$ structures we will give a direct proof of the equivalence of these two notions (not using the Cartan-Kaehler theorem) in a paper to follow this one.

Finally we mention that the following are known for $C^{\infty} G$-structures:

Received by the editors April 13, 1964.

${ }^{1}$ ) This work has been partially supported by the National Science Foundation under Grant NSF GP 1904. 
Formal integrability implies integrability if the linear group, $G$, is completely reducible (this is a result of the work of Sternberg and Singer on the infinite Lie groups [cf. [10]]) or if one of the prolongations of $G$ is of "elliptic type" (cf. Spencer [11]).

1. Algebraic preliminaries. In this chaper $g$ will always stand for a linear L.A., that is, given a vector space $V, g$ will be a subalgebra of $g L(V)$. We are going to associate with $g$ a certain chain complex, the so-called $\delta$ complex of Spencer. The homology theory based on this chain complex will figure centrally in our theory of integrability of $G$-structures.

Given $G$, we define $g^{(1)}$ to be the set of all linear mappings $S: V \rightarrow g$ such that for all $v$ and $w$ in $V, S \circ v$ applied to $w$ equals $S \circ w$ applied to $v$.

Having defined $g^{(k-1)}$ by recurrence, we define $g^{(k)}$ to be the set of all linear mappings $S: V \rightarrow g^{(k-1)}$ such that, for all $v$ and $w$ in $V, S \circ v$ applied to $w$ equals $S$ o $w$ applied to $v$. Note that this makes sense since $g^{(k-1)}$ is contained in $\operatorname{Hom}\left(V, g^{(k-2)}\right)$ by recurrence.

The definition of $g^{(k)}$ can be written more succinctly:

$$
g^{(k)}=g \otimes S^{k}\left(V^{*}\right) \cap V \otimes S^{k+1}\left(V^{*}\right)
$$

$g^{(k)}$ also has a simple analytical interpretation which will be described in $\$ 3$.

Let $v$ be an arbitrary element of $V$. $v$ can be thought of as a linear functional on $V^{*}$ and can be extended uniquely to a derivation on $S\left(V^{*}\right)$ (cf. Chevalley [4]). We will denote this by $D_{v}$.

Let $v_{1} \otimes s_{1}$ be an element of $V \otimes S^{k+1}\left(V^{*}\right)$ and $v_{2} \otimes s_{2}$ an element of $V \otimes S^{l+1}\left(V^{*}\right)$. We will define the bracket of these two elements (denoted by the symbol "[ ]") by the expression

$$
v_{2} \otimes D_{v_{1}} s_{2} \circ s_{1}-v_{1} \otimes D_{v_{2}} s_{1} \circ s_{2}
$$

Note that this lies in $V \otimes S^{k+l+1}\left(V^{*}\right)$.

"[ ]" extends to a bilinear mapping of $V \otimes S^{k+1}\left(V^{*}\right) \times V \otimes S^{l+1}\left(V^{*}\right)$ into $V \otimes S^{k+l+1}\left(V^{*}\right)$. Recalling (1.1), this induces a bilinear mapping of $g^{(k)} \otimes g^{(l)}$ into $V \otimes S^{k+l+1}$, which is in fact a bilinear mapping into $g^{(k+h)}$. Moreover "[ ]" makes the vector space

$$
V+g+g^{(1)}+\cdots \text { (infinite sum) }
$$

into a Lie algebra. Both of these remarks will be obvious in light of $\$ 3$.

Consider the complex $\sum C^{k, l}=C^{\#}$, where

$$
C^{k, l}=S^{k}\left(V^{*}\right) \otimes \bigwedge^{\prime}\left(V^{*}\right) .
$$

We will define on $C^{\#}$ and antiderivative $\delta$ of bidegree $(-1,1)$.

By requiring $\delta$ to be an antiderivative we only have to define it on the elements of $C^{-, 0}$ and $C^{-, 1}$ : 
Let $s$ be in $S^{k}\left(V^{*}\right)$. For each $v$ in $V, D_{v} s$ is in $S^{k-1}\left(V^{*}\right)$; therefore, $s$ defines an element of $\operatorname{Hom}\left(V, S^{k-1}\left(V^{*}\right)\right)$ or $C^{k-1,1}$. Denote this by $\delta s$. Let $s$ be in $C^{k, 1}$. We can regard it as an element of $\operatorname{Hom}\left(V, S^{k}\left(V^{*}\right)\right)$. For any pair of elements, $v$ and $w$, in $V$, we set

$$
\langle\delta s, v \wedge w\rangle=D_{v}(s \circ w)-D_{w}(s \circ v) .
$$

$\delta s$ is in $\operatorname{Hom}\left(V \wedge V, S^{k-1}\left(V^{*}\right)\right)$ or $C^{k-1,2}$.

The definition of $\delta$ now extends without any trouble to all of $C^{\#}$.

An elementary computation shows that $\delta^{2}=0$; and it is not much harder to show that the homology theory based on $\left(C^{*}, \delta\right)$ is trivial. (This is the formal analogue of the Poincare Lemma. Cf. Spencer [11].)

We can extend $\delta$ to the chain complex $V \otimes C^{*}\left(V^{*}\right)$ in the standard way by letting it act trivially on $V$. Let

$$
C^{k, l}(g)=g^{k-1} \otimes \bigwedge^{l}\left(V^{*}\right) .
$$

Because of the identification (1.1), the sum $\sum C^{k, l}(g)$ can be thought of as a subspace of $V \otimes C^{\#}\left(V^{*}\right)$ and it is evidently even a subcomplex.

The homology theory based on this bigraded chain complex will be called the Spencer $\delta$-cohomology of $g$. It is a consequence of the definitions that

$$
H^{i, 1}(g)=0 \quad \text { for } i \geqq 1,
$$

and

$$
H^{i, j}(g)=0 \text { for } i<0 .
$$

We will be mainly interested in the homology groups $H^{i, 2}(g)$. Our obstructions to integrability will be tensors taking their values in these homology groups; therefore if certain of these homology groups vanish, we have $a$ priori information about flatness. It is a result of Spencer's that for $i$ sufficiently large the $H^{i, j}(g)$ all vanish, which implies that the problem of determining whether a G-structure is (formally) flat can always be settled in a finite number of steps.

2. $G$-structures. In this chapter $V$ will be an $n$-dimensional vector space, $G$ a Lie subgroup of $G L(V)$, and $g$ its associated Lie algebra. Let $M$ be an $n$-dimensional differentiable $\left({ }^{2}\right)$ manifold. A $G$-structure on $M$ is a law which assigns to each point, $P$, of $M$ a set, $\epsilon_{P}$, of linear mappings of $V$ onto the tangent space of $M$ at $P$ such that:

I. If $A$ is in $\epsilon_{P}$ and $B$ is in $\epsilon_{P}, A^{-1} B$ is in $G$.

II. If $A$ is in $\epsilon_{P}$ and $B$ is in $G, A B$ is in $\epsilon_{P}$.

In addition it is assumed that $\epsilon_{P}$ depends differentiably on $P$, or, in other words, that

(2) Throughout this paper, differentiable will always mean $C^{\infty}$. 


$$
E=\bigcup_{P \in M} \epsilon_{P}
$$

admits local differentiable cross-sections. The elements of $E$ are called the "frames" of $E$.

If we let $\epsilon_{P}$ be the set of all linear mappings of $V$ onto the tangent space at $P$, we get a $G L(V)$-structure on $M$, the so-called principal frame bundle, which we will denote by $F$. A $G$-structure on $M$ is simply a reduction of $F$ to a principal subbundle with group $G$.

Let $M$ and $M^{\prime}$ be two $n$-dimensional manifolds and let $f$ be a diffeomorphism of $M$ into $M^{\prime}$.

At each point, $P$, of $M$ it defines a mapping $f_{\#}: T_{P} \rightarrow T_{f(P)}$.

If $A$ is in $\operatorname{Hom}\left(V, T_{P}\right), f_{\#} \circ A$ is in $\operatorname{Hom}\left(V, T_{f(P)}\right)$; therefore, $f$ induces a mapping, $\tilde{f}$, of the principal frame bundle on $M$ into the principal frame bundle on $M^{\prime}$. Suppose that $M$ and $M^{\prime}$ both possess $G$-structures. If $\tilde{f}$ maps the frames of one into the frames of the other, $f$ is called structure preserving.

With this definition in mind it is intuitively clear what is meant by a mapping of $M$ into $M^{\prime}$ which is structure preserving to kth order at some point of $M$. We must, however, give this notion a precise meaning:

Let $M$ be a differentiable manifold and let $N_{1}$ and $N_{2}$ be two $m$-dimensional submanifolds of $M$ intersecting at the point $P$.

We say that $N_{1}$ and $N_{2}$ have $k$ th order contact at $P$ if we can find a coordinate system for $M,\left(y_{1}, \cdots, y_{n}\right)$, defined in a neighborhood of $P$, such that the equations of $N_{1}$ are given by

$$
y_{m+1}=0, y_{m+2}=0, \cdots, y_{n}=0,
$$

and the equations of $N_{2}$ by

$$
\begin{array}{cc}
y_{m+1} & =f_{m+1}\left(y_{1}, \cdots, y_{n}\right), \\
\vdots & \vdots \\
y_{n} & =f_{n}\left(y_{1}, \cdots, y_{n}\right),
\end{array}
$$

where $f_{m+1}, f_{m+2}, \cdots, f_{n}$ have vanishing derivatives of all orders less than or equal to $k$ at the point $P$.

Now let $M$ and $M^{\prime}$ be two $n$-dimensional manifolds; let $E \rightarrow M$ be a $G$ structure on $M$; let $E^{\prime} \rightarrow M^{\prime}$ be a $G$-structure on $M^{\prime}$, and let $F$ and $F^{\prime}$ be the principal frame bundles on $M$ and $M^{\prime}$. Let $P$ be a point of $M$ and $f$ a diffeomorphism of a neighborhood of $P$ into $M^{\prime}$. We will say that $f$ preserves the G-structure to kth order at $P$ if some frame belonging to $E$ at $P$ gets mapped by $\tilde{f}$ onto a frame, $A$, belonging to $E^{\prime}$ and if $\tilde{f}(E)$ and $E^{\prime}$ have contact of order $k$ at $A$ as submanifolds of $F^{\prime}$.

Note that this notion does not depend on $f$ itself but only on the $(k+1)$ jet of $f$ at $P$. Therefore it makes sense to say that a $(k+1)$-jet with source $P$ and target $Q$ is structure preserving to order $l, l \leqq k$, at $P$ and $Q$. 
Note also that the composition of two $(k+1)$-jets which are structure preserving to order $k$ is structure preserving to order $k$. Likewise the inverse of $\mathrm{a}(k+1)$-jet which is structure preserving to order $k$ is structure preserving to order $k$.

3. Properties of integrable $G$-structures. If we think of $V$ as a differentiable manifold, it posssesses a special $G$-structure defined as follows: At each point, $P$, of $V$, there exists a canonical isomorphism

$$
d P: V \rightarrow T_{P} .
$$

The $G$-structure in question can be written explicitly as the set of all linear mappings:

$$
d P \circ A, \quad P \in V, A \in G .
$$

Any $G$-structure which is locally equivalent to this $G$-structure is said to be integrable or flat $\left({ }^{3}\right)$. We will denote it by $E_{0}$. Also we will denote the principal frame bundle on $V$ by $F_{0}$. In addition we will consider the following objects:

(1) $E_{0}^{k}$ the set of all $(k+1)$-jets with source at the zero element of $V$ and target anywhere in $V$, which are structure preserving to order $k$.

(2) $F_{0}^{k}$ the set of all inversible $(k+1)$-jets with source at the zero element of $V$ and target anywhere in $V$.

(3) $G^{k}$ the set of all $(k+1)$-jets with source and target at the zero element of $V$ which are structure preserving to order $k$.

It follows from the remarks at the end of $\$ 2$ that $G^{k}$ is a group, and that $E_{0}^{k}$ is a principal fiber bundle over $V$ with structural group $G^{k}$. (Note that $G^{k}$ acts on the right.)

In $\$ 4$ we will need a more explicit description of $G^{k}$. We must first of all, therefore, write out what its algebra is.

Let $x_{1}, \ldots, x_{n}$ be a system of linear coordinates in $V$ and let $f(t)$ be a $(k+1)$-jet belonging to $G^{k}$, and depending differentiably on $t$. We will also assume that $f(0)$ is the $(k+1)$-jet of the identity mapping.

There exists a representative of $f(t)$ of the form:

$$
y_{i}=a_{i}^{j}(t) x_{j}+\cdots+a_{i}^{j_{1} \cdots j_{k+1}}(t) x_{j_{1}} \cdots x_{j_{k+1}} .
$$

$\tilde{f}$ is represented by the matrix

$$
\frac{\partial y_{i}}{\partial x_{j}}=a_{i}^{j}(t)+\cdots+\frac{\partial}{\partial x^{a}} a_{i}^{j_{1} \cdots j_{k+1}} x_{j_{1}} \cdots x_{j_{k+1}} .
$$

To $k$ th order this must be the form $b_{i}^{j}\left(x_{1}, \cdots, x_{n}, t\right)$, where $\left(b_{i}^{j}\left(x_{1}, \cdots, x_{n}, t\right)\right)$ is a matrix in G. Differentiating (3.1) with respect to $t$ we get the following conditions on the coefficients of $f$.

(3) Equivalently this means that one can introduce coordinates $\left(x_{1}, \cdots, x_{n}\right)$ locally such that $\left(\partial / \partial x^{1}, \cdots, \partial / \partial x^{n}\right)$ is a local cross-section of the $G$-structure. 


$$
\begin{gathered}
\frac{d}{d t} a_{j}^{i}(0) \in g, \\
\frac{d}{d t} a_{i}^{j_{1} \cdots j_{l+1}(0) \frac{\partial}{\partial x^{x}} x_{j_{1}} \cdots x_{j_{l+1}}} \in V \otimes S^{l+1}\left(V^{*}\right) \cap g \otimes S^{l}\left(V^{*}\right)
\end{gathered}
$$

for $l \leqq k$.

In other words, as a vector space, the Lie algebra of $G^{k}$ is a direct sum of $\left({ }^{4}\right)$

$$
g+g^{(1)}+\cdots+g^{(k)} .
$$

It is not hard to show in addition that the bracket operation on the Lie algebra of $G^{k}$ coincides with the bracket operation already defined on

$$
g+g^{(1)}+g^{(2)}+\cdots+g^{(k)}+g^{(k+1)}+\cdots
$$

truncated at degree $k$.

The group $G^{k}$ itself is now easy to define abstractly as a semidirect product of $G$ and the nilpotent Lie group generated by

$$
g^{(1)}+g^{(2)}+\cdots+g^{(k)}+\cdots / g^{(k+1)}+\cdots
$$

We will, however, omit details.

For each $m>0$ we will denote by $i_{m}$ the $(m+1)$-jet of the identity mapping of $V$ onto itself with source and target at the zero element. Let $\phi$ be an element of $G^{k}, \phi^{\prime}$ its image in $G^{k-1}$ and $f$ a mapping of $V$ into $V$ having $\phi$ as its $(k+1)$-jet. It is clear that there exists a neighborhood of $\phi^{\prime}$ in $E_{0}^{k-1}$ such that if $\psi$ is in this neighborhood the composition, $f^{-1} \circ \psi$, is defined.

The correspondence

$$
\psi \rightarrow f^{-1} \circ \psi
$$

defines on this neighborhood a mapping of $E_{0}^{k-1}$ into $F_{0}^{k-1}$ which maps $\phi^{\prime}$ onto $i_{k-1}$. The 1-jet of this mapping depends only on $\phi$. Moreover, this one jet defines a mapping of the tangent space to $E_{0}^{k-1}$ at $\phi^{\prime}$ onto the tangent space to $E_{0}^{k-1}$ at $i_{k-1}$.

On the other hand $\phi^{\prime}$ regarded as an element of $G^{k-1}$ operates on $E_{0}^{k-1}$ on the right, sending $i_{k-1}$ onto $\phi^{\prime}$ and the tangent space at $i_{k-1}$ onto the tangent space at $\phi^{\prime}$. If we compose this mapping with the mapping just described we get a mapping of the tangent space at $i_{k-1}$ onto itself. In other words, we have a natural representation of $G^{k}$ on the tangent space to $E_{0}^{k-1}$ at $i_{k-1}$. We will denote this representation by $\rho_{k}$.

We can write it out explicitly in terms of $V+g+\cdots+g^{(k-1)}$, though we will not bother to do so here.

(4) Note that this identification does not depend on the choice of linear coordinates in $V$. 


\section{4. $G$-structures which are uniformly $k$-flat.}

Definition. A $G$-structure on the manifold $M$ will be called uniformly $k$-flat if at each point, $P$, of $M$ there exists a $(k+1)$-jet with target at $P$ and source at the zero element in $V$ which is $k$ th order structure preserving $\left({ }^{5}\right)$.

Let the given $G$-structure be defined by the fibering

$$
\boldsymbol{E} \rightarrow \boldsymbol{M} \text {. }
$$

We define $E^{k}$ to be the set of all $(k+1)$-jets with source at the zero element in $V$ and target at some point of $M$, which are $k$ th order structure preserving.

$E^{k}$ is a fiber bundle over $M$ with structural group $G^{k}$ (acting on the right). We will briefly describe some of its intrinsic properties:

Let $\phi$ be an element of $E^{k}, \phi^{\prime}$ its image in $E^{k-1}$ and $f$ a mapping of $V$ into $M$ whose $(k+1)$-jet is $\phi$. There exists a neighborhood of $\phi^{\prime}$ in $E^{k-1}$ such that if $\phi$ is an element of this neighborhood $f^{-1} \circ \psi$ is well defined. The correspondence

$$
\psi \rightarrow f^{-1} \circ \psi
$$

defines a mapping of this neighborhood into $F_{0}^{k-1}$ which maps $\phi^{\prime}$ onto $i_{k-1}$. The one jet of this mapping depends only on $\phi$ and maps the tangent space to $E^{k-1}$ at $\phi^{\prime}$ onto the tangent space to $E_{0}^{k-1}$ at $i_{k-1}$; therefore, it can be regarded as a one form, defined at $\phi^{\prime}$, with values in the vector space

$$
V+g+\cdots+g^{(k-1)} \text {. }
$$

If we lift this form to $\phi$ we get a canonically defined one-form at $\phi$. This is true for all elements of $E^{k}$, so we have a canonically defined oneform on $E^{k}$ with values in (4.1). We will denote this by $\Gamma^{k}$. We will also denote its homogeneous parts by $\omega, \Omega^{0}, \Omega^{1}, \ldots, \Omega^{k-1}$.

The transformation properties of $\Gamma^{k}$ are easy to describe in terms of the representation, $\rho_{k}$, of $G^{k}$ on $V+g+\cdots+g^{k-1}$ described in the last section. If $s$ is an element of $G^{k}$ it acts on the right on $E^{k}$ and thus defines a diffeomorphism, $\bar{s}$, of $E^{k}$ on itself. One verifies immediately that

$$
\bar{s}^{*} \Gamma^{k}=\rho_{k}\left(s^{-1}\right) \Gamma^{k} \text {. }
$$

We will now define on $E$ a structural invariant which resembles very much the Ehresmann-Bernard structure tensor and in fact reduces to it if $k=0$.

To do so, let $\phi$ be an element of $E^{k}$ and $P$ its projection on $M$.

Let $H$ be a horizontal subspace of the tangent space to $E^{k}$ at $\phi$ on which the forms $\Omega^{0}, \Omega^{1}, \ldots, \Omega^{k-1}$ all vanish. Such a subspace always exists but in general is not unique.

(5) The $G$-structures in question being of course the given $G$-structure and the integrable $G$-structure defined in the previous section. 
Now let $v$ and $w$ be elements of $V$, and $\bar{v}$ and $\bar{w}$ the corresponding tangent vectors to $V$ at the zero element. $(\phi)_{*} \bar{v}$ and $(\phi)_{*} \bar{w}$ are tangent to $M$ at $P$, and determine uniquely vectors $X$ and $Y$ in $H$ projecting onto them. Define

$$
\Pi_{H}(v, w)=\left\langle d \Gamma^{k}, X \wedge Y\right\rangle .
$$

$\Pi_{H}$ is a linear mapping of $V \wedge V$ into $V+g+\cdots+g^{(k-1)}$. We will denote the $i$ th homogeneous part of this mapping by $\Pi_{H}^{(i)}$.

Proposition 4.1. $\Pi_{H}^{(i)}=0, i<k-1$.

Proof. Let $X$ and $Y$ be two vectors which are tangent to $E^{k}$ at $\phi$ and denote their projections on $H$ by $X^{\prime}$ and $Y^{\prime}$. We will define the two-form $d \Gamma^{k} \circ H$ by the relation

$$
\left\langle d \Gamma^{k} \circ H, X \wedge Y\right\rangle=\left\langle d \Gamma^{k}, X^{\prime} \wedge Y^{\prime}\right\rangle .
$$

$d \Omega^{i} \circ H$ will be similarly defined.

The proposition amounts to showing that $d \Omega^{i} \circ \mid H=0, i<k-1$.

We will first show that, for $i<k-1$, the following identities hold:

$$
d \Omega^{i} \circ H=d \Omega^{i}+\frac{1}{2}\left(\left[\omega, \Omega^{i+1}\right]+\left[\Omega^{0}, \Omega^{i}\right]+\cdots+\left[\Omega^{i+1}, \omega\right]\right) .
$$

First of all notice that both the left- and right-hand sides are horizontal, that is, their inner product with a vertical vector vanishes. Next, if $X$ and $Y$ both belong to $H$, the left-hand side applied to $X \wedge Y$ equals $\left\langle d \Omega^{i}, X \wedge Y\right\rangle$ and the right-hand does also, since $\Omega^{0}, \Omega^{1}, \ldots, \Omega^{i+1}$ vanish on $H$. This establishes the identity.

Now let $\Gamma_{0}^{k}$ be the structure form on $E_{0}^{k}$. Indicate its components by $\omega_{0}, \cdots, \Omega_{0}^{k-1}$. It is easy to show that, for $i<k-1$

$$
d \Omega_{0}^{i}+\frac{1}{2}\left(\left[\omega_{0}, \Omega_{0}^{i+1}\right]+\cdots+\left[\Omega_{0}^{i+1}, \omega_{0}\right]\right)=0
$$

(the equations of Maurer-Cartan).

Finally note that the forms $\omega, \cdots, \Omega^{k-1}$ are obtained from the forms $\omega_{0}, \cdots, \Omega_{0}^{k-1}$ in the following way. As we have already shown, $\phi$ defines a mapping $\bar{\phi}$ of the tangent space to $E^{k-1}$ at $\phi^{\prime}$ onto the tangent space to $E_{0}^{k-1}$ at $i_{k-1}$. At $i_{k-1}$ the form $\Gamma_{0}^{k}$ (which takes its values in the tangent space to $E_{0}^{k-1}$ at $i_{k-1}$ ) is simply the identity map of the tangent space to $E_{0}^{k-1}$ at $i_{k-1}$ onto itself. By definition $\Gamma^{k}=\bar{\phi}^{*} \Gamma_{0}^{k}$ at $\phi^{\prime}$.

Also, for $i<k-1, \Omega^{i}$ is globally defined on $E^{k-1}$ and $\Omega_{0}^{i}$ is globally defined on $E_{0}$. It is easy to show that $d \Omega^{i}=\bar{\phi}^{*} d \Omega_{0}^{i}$ at $\phi^{\prime}$. It follows that the equation (4.4) implies the vanishing of (4.3). Q.E.D.

The term $\Pi_{H}^{(k-1)}$ will not in general be equal to zero. We want to determine, however, to what extent it depends on $H$. Let us first of all differentiate the right-hand side of (4.3) with $i=k-2$. After a simple calculation we obtain: $\left[d \Omega^{k-1} \circ H, \omega\right]=0$. 
Expressed in terms of $\Pi_{H}^{(k-1)}$ this simply says that $\delta \Pi_{H}^{(k-1)}=0$.

Thus we have established:

Proposition 4.2. $\Pi_{H}^{(k-1)}$ is a cocycle of bidegree $(k, 2)$ in the chain complex $C^{\#}$ (g) (cf. 81, (1.2)).

We will now prove:

Proposition 4.3. The cohomology class of $\Pi_{H}^{(k-1)}$ in $H^{k, 2}(g)$ is independent of the choice of the horizontal space $H$.

Proof. We must show that another choice of $H$ only changes $\Pi_{H}^{(k-1)}$ by a coboundary. To show this, let $H^{\prime}$ be another choice of a horizontal subspace. Associated with $H$ and $H^{\prime}$ we get an element, $s_{H, H^{\prime}}$, of $g^{(k)} \otimes V^{*}$ defined as follows: As above, for each $v$ in $V$, we denote the tangent vector it defines at the zero element of $V$ by $\bar{v} . \phi_{*} \bar{v}$ is tangent to $M$ at $P$ and has a unique lift, $X$, to $H$, and a unique lift, $X^{\prime}$, to $H^{\prime} . X-X^{\prime}$ is tangent to the fiber and can be thought of as being in the Lie algebra of $G^{*}$. In fact, it lies in $g^{(k)}$ since both $H$ and $H^{\prime}$ are annihilated by $\Omega^{0}, \cdots, \Omega^{k-1}$. We set $s_{H, H^{\prime}}(v)$ equal to this element of $g^{(k)}$.

We will show

$$
\Pi_{H^{\prime}}^{(k-1)}-\Pi_{H}^{(k-1)}=\delta s_{H, H^{\prime}}
$$

Let $v$ and $w$ be in $V$; let $X$ and $Y$ be vectors in $H$ which project onto $\phi_{*} \bar{v}$ and $\phi_{*} \bar{w}$, and let $X^{\prime}$ and $Y^{\prime}$ be the corresponding vectors in $H^{\prime}$. Let $X^{\prime}-X=\alpha$ in $g^{(k)}$ and $Y^{\prime}-Y=\beta$.

$$
\begin{aligned}
\left\langle d \Gamma, X^{\prime}\right. & \left.\wedge Y^{\prime}\right\rangle-\langle d \Gamma, X \wedge Y\rangle \\
& =\left\langle d \Gamma,\left(X^{\prime}-X\right) \wedge Y^{\prime}\right\rangle+\left\langle d \Gamma, X \wedge\left(Y^{\prime}-Y\right)\right\rangle \\
& \left.\left.=(\alpha\lrcorner d \Gamma^{k}\right)\left(Y^{\prime}\right)-(\beta\lrcorner d \Gamma^{k}\right)(X) .
\end{aligned}
$$

But by (4.2) we obtain

$$
\left.(\alpha\lrcorner d \Gamma^{k}\right)\left(Y^{\prime}\right)=\mathscr{L}_{\alpha} \Gamma^{k}\left(Y^{\prime}\right)=\alpha \circ w .
$$

Similarly we get

$$
\left.(\theta\lrcorner d \Gamma^{k}\right)(X)=\beta \circ v \text {. }
$$

Therefore

$$
\Pi_{H^{\prime}}(v, w)-\Pi_{H}(v, w)=\alpha \circ w-\beta \circ v .
$$

However, by definition, $\alpha=s_{H, H^{\prime}}(v)$ and $\beta=s_{H, H^{\prime}}(w)$, so we get (4.5) as claimed.

We will denote by $c^{k}$ the mapping of $E^{k}$ into $H^{k, 2}(g)$ which we have just defined. Its transformation properties are easy to write down from (4.2). It is constant on the fibers of the fibration $E^{k} \rightarrow E$; therefore it is actually defined on $E$, not on $E^{k}$. On $E$ it transforms like a tensor of type $H^{k, 2}(g)$. We will call $c^{k}$ the $k t h$ structure tensor of $E$. 
Our basic theorem is the following.

Theorem 4.1. Suppose $E \rightarrow M$ is uniformly $k$-flat. Let $P$ be a point of $M$. If the kth structure tensor vanishes at $P$ there exists $a(k+2)$-jet with source at the zero element of $V$ and target at $P$, which is structure preserving to $(k+1)$ st order. The converse is also true.

Corollary. Let $E \rightarrow M$ be uniformly $k$-flat. It is uniformly $(k+1)$-flat if and only if its kth structure tensor vanishes.

COROLLARY. If $H^{k, 2}(g)=0$, every G-structure which is uniformly $k$-flat is uniformly $(k+1)$-flat.

Proof of the theorem. Let $\phi$ be an element of $E^{k}$ projecting on $P$. We first remark that the vanishing of $c^{k}$ at $P$ implies that there exists a oneform, $\Omega^{k}$, defined at $\phi$, with values in $g^{(k)}$ such that the equation

$$
d \Omega^{k-1}+\frac{1}{2}\left(\left[\omega, \Omega^{k}\right]+\left[\Omega^{0}, \Omega^{k-1}\right]+\cdots+\left[\Omega^{k}, \omega\right]\right)=0
$$

is satisfied, and such that if $X$ is an element of the Lie algebra of $G^{k}$ and $\widetilde{X}$ is the corresponding vector tangent to the fiber at $\phi, \Omega^{k}(\widetilde{X})$ is the $g^{(k)}$ component of $X$.

The one-form $\Omega=\left(\omega, \Omega^{0}, \cdots, \Omega^{k}\right)$ defines a mapping, $\gamma$, of the tangent space to $E^{k}$ at $\phi$ onto the tangent space to $E_{0}^{k}$ at $i_{k}$. By defining it properly on the vectors tangent to the fiber, we extend this mapping to a mapping, $\bar{\gamma}$, which maps the tangent space to $F^{k}$ at $\phi$ onto the tangent space to $F_{0}^{k}$ at $i_{k}$. The system of equations for $\bar{\gamma}$ analogous to those of (4.6) is simply the condition that $\bar{\gamma}$ be induced by a $(k+2)$-jet of a mapping of $\boldsymbol{M}$ into $V$ mapping $P$ onto the zero element. To prove this we notice that by Proposition 4.1 every such $(k+2)$-jet induces a 1 -jet satisfying these equations and that the number of $(k+2)$-jets extending a given $(k+1)$-jet is equal to the number of solutions of these equations (i.e. $\operatorname{dim} V \otimes S^{k+2}\left(V^{*}\right)$ ). We thus establish that $\bar{\gamma}$ comes from the $(k+2)$-jet of a mapping, $f$, of $M$ into $V$. However, by definition, $\bar{\gamma}$ maps the space tangent to $E^{k}$ at $\phi$ onto the space tangent to $E_{0}^{k}$ at $i_{k}$. This implies that $f$ is structure preserving at $P$ to order $k+1$. The converse can be proved by exactly the same argument in reverse order, so we will omit details.

5. $G$-structures of finite type.

Definition. A $G$-structure will be said to be of finite type if $g^{(k)}=0$ for some $k$. If $g^{(k-1)} \neq 0$ and $g^{(k)}=0, G$ will be said to be of type $k$.

It can be proved (cf. \$6) that a subgroup of $G L(V)$ which leaves fixed a positive definite quadratic form on $V$ is of type 1 . Since every compact subgroup of $G L(V)$ satisfies this requirement, every linear representation of a compact group is of type 1 . In fact Cartan has shown that most completely reducible linear representations are of type 1 . The exceptional 
cases are listed by Cartan in [2].

Note that if $G$ is of type $k, H^{r, 2}(G)=0$ for $r>k$.

We will prove for finite $G$-structures the following integrability theorem.

TheOREM 5.1. If $G$ is of type $k, a$ G-structure which is uniformly $(k+1)$ flat is integrable.

Proof. First of all, by the main theorem of the last section, a $G$-structure which is uniformly $(k+1)$-flat is uniformly $(k+2)$-flat since $H^{k+1,2}(g)=0$. Since $g^{(k)}=g^{(k+1)}=0$, the fiber bundles $E^{k}, E^{k+1}$, and $E^{k+2}$ are identical. Therefore the structure forms $\Gamma^{k+2}$ and $\Gamma^{k+1}$ are defined on the same manifold, i.e. $E^{k}$, and are identical. It is easy to establish the following properties for $\Gamma^{k+1}$ :

A. If $X$ is tangent to $E^{k}$ at some point and $\Gamma^{k+1}(X)=0$, then $X=0$; that is, the components of $\Gamma^{k+1}$ give rise to a complete parallelism on $E^{k}$.

B. If $X$ is a vector in the Lie algebra of $G^{k}$ and $\widetilde{X}$ is the vector field it induces on $E^{k}$, then $\Gamma^{k+1}(\widetilde{X})=X$.

C. $d \Gamma^{k+1}+\frac{1}{2}\left[\Gamma^{k+1}, \Gamma^{k+1}\right]=0$ (by Proposition 4.1 applied to $\Gamma^{k+2}$ ).

We will denote by $\Gamma_{0}^{k+1}$ the analogous form on $E_{0}^{k}$. Given an arbitrary point of $E^{k}$ and an arbitrary point of $E_{0}^{k}$, we can demonstrate the existence of a unique mapping $f, E^{k} \rightarrow E_{0}^{k}$ mapping the one point onto the other and satisfying

$$
f^{*} \Gamma_{0}^{k+1}=\Gamma^{k+1} \text {. }
$$

(Apply Frobenius' theorem to A, C, and the analogous statements for $\Gamma_{0}^{k+1}$.) Condition $B$ implies that this is a fiber mapping. It therefore induces a mapping

$$
g: M \rightarrow V
$$

and a fiber mapping

$$
g^{\prime}: E \rightarrow E_{0}
$$

lifting $g$. From equation (5.2) one gets

$$
\left(g^{\prime}\right)^{*} \omega_{0}=\omega,
$$

which implies that $g^{\prime}=g$ (cf. $\$ 2$ ). Therefore $g$ is structure preserving. Q.E.D.

\section{Examples.}

A. Let $G=O(n)$, the orthogonal group in dimension $n$. It is easy to show that giving an $O(n)$-structure on a manifold $M$ is equivalent to giving a Riemannian metric on $M$. One can also verify without too much trouble that $g^{(1)}=0$ and $H^{0,2}(g)=0$ (cf. [10]). Therefore $O(n)$ is of type 1 and the only obstruction to integrability lies in $H^{1,2}$. We will show in $\$ 8$ that this obstruction is the ordinary Riemannian curvature of $M$.

B. Let $G=O(n)+R$, the conformal group on $R^{n}$. A $G$-structure on 
$M$ is a conformal structure on $M$ (i.e., an intrinsically defined notion of angle). One can show (cf. [10] again) that $g^{(2)}=0$ and $g^{(1)} \approx R^{n^{*}}$ providing $n>2$. (If $n=2, g$ is of infinite type.) As far as the homology goes, if $n=3, H^{i, 2}=0$ except when $i=2$, and if $n>3, H^{i, 2}=0$ except when $i=1$. This fact explains some of the formal differences between conformal geometry in three dimensions and conformal geometry in more than three dimensions. In the first case the obstruction to integrability lies in $H^{2,2}$ and is the classical conformal tensor of Shouten-Weyl [9]. In the second case the obstruction lies in $H^{1,2}$ and is the Weyl conformal curvature tensor [13].

C. Let $G=G L(C, n)$, the complex linear group on $C^{n}=R^{2 n}$. A $G$ structure on $M$ is in this case an almost complex structure on $M$. One can show that $G$ is of infinite type, and that $H^{i, j}=0$ for all $i>0$ and all $j$. The only obstruction to (formal) integrability lies in $H^{0,2}$ and is the classical structure tensor for almost complex structures. A well-known theorem of Newlander-Nirenberg states that the vanishing of this tensor implies integrability.

D. Involutive groups. A represented group is said to be involutive if $H^{i, j}=0$ for all $i>0$ and all $j$. (The classical definition of involutiveness is formulated in a rather different way (cf. Cartan [2] and Matsushima [7]). Serre has shown this definition is equivalent to the one just given (cf. [8]).)

$G L(C, n)$ is an example of an involutive group; and, more generally, if $A$ is any $n \times n$ matrix, the set of all nonsingular matrices commuting with $A$ is an involutive group. Other examples are the simplectic group and the group associated with a multifoliate structure.

If the structure group of a $G$-structure is involutive the only obstruction to (formal) integrability lies in $H^{0,2}$ and is simply the Ehresmann-Bernard structure tensor. It is known that the vanishing of this tensor implies integrability in all of the special cases just mentioned.

E. Let $p(x)$ be a nondegenerate homogeneous polynomial in $n$ indeterminates. (This means that $p(x)$ cannot be reduced to a polynomial in less than $n$ indeterminates by a linear change of variables.) Let $G$ be the group of linear transformations of the $n$-variables which leave $p(x)$ fixed. Singer and I will show in a forthcoming paper that $G$ is always of finite type. (In fact of type $\leqq K$ if $p(x)$ is of degree $K$.) We have conjectured that $G$ is actually of type 1 . This is an interesting fact to know in connection with the following question. When can a linear differential operator be transformed into a constant coefficient operator by a change of coordinates?

\section{APPENDIX}

7. Cartan connections. In a series of classical papers Cartan showed that 
the study of certain kinds of geometries gives rise naturally to connections, for example, in Riemannian geometry, the connection of Levi-Civita. In this section we will explain how such connections arise in studying $G$ structures of finite type.

Let $G$ be a subgroup of $G L(V)$ of type $k$. The algebra

$$
V+g+g^{(1)}+\cdots
$$

is finite-dimensional since $g^{(k)}=g^{(k+1)}=\cdots=0$, and its underlying space is

$$
V+g+\cdots+g^{(k-1)} \text {. }
$$

We have shown that

$$
g+g^{(1)}+\cdots+g^{(k-1)}
$$

is the Lie algebra of $G^{k-1}$. In the discussion below $H$ will be a Lie group whose Lie algebra is $V+g+g^{(1)}+\cdots+g^{(k-1)}$, such that $G^{k-1}$ is the subgroup of $H$ corresponding to the subalgebra $g+\cdots+g^{(k-1)}$.

In $\$ 5$ we showed that a $G$-structure which is uniformly $(k+1)$-flat is integrable. We will now consider $G$-structures which are uniformly $k$-flat.

Since $g^{(k)}=0, G^{k}=G^{k-1}$ and the representation $\rho_{k}$, of $G^{k}$ on $V+g+$ $\cdots+g^{(k-1)}$ reduces to the adjoint representation. Moreover $E^{k}=E^{k-1}$, and the structure form of $E^{k}, \Gamma^{k}$, is defined on $E^{k-1}$. One easily verifies the following properties of $\Gamma^{k}$ (compare with \$5).

A. If $X$ is tangent to $E^{k-1}$ at some point and $\Gamma^{k}(X)=0$, then $X=0$; that is, the components of $\Gamma^{k}$ give rise to a complete parallelism on $E^{k}$.

B. If $X$ is a vector in the Lie algebra of $G^{k-1}$ and $\widetilde{X}$ is the vector field it induces on $E^{k-1}$ then $\Gamma^{k}(\widetilde{X})=X$.

C. If $s \in G^{k-1}, \bar{s}^{*} \Gamma^{k}=\operatorname{Ad}^{-1} \Gamma^{k}$ (cf. equation (4.2)).

In the product space $E^{k-1} \times H$ consider the following relation:

$$
(x, t) \sim\left(x^{\prime}, t^{\prime}\right)
$$

if and only if there exists an $s$ in $G^{k-1}$ such that $x^{\prime}=x s$ and $t^{\prime}=s^{-1} t$. Denote by $E$ the set of equivalence classes of this relation. $E$ is a fiber bundle over $M$ with structural group $H$. Moreover if we associate with every element $x$ of $E$ the equivalence class of the couple $\left(x, i_{k-1}\right)$ we get an inclusion of $E^{k-1}$ into $E$. We now extend the form $\Gamma^{k}$ to $E$. To do so, we first define it at every point of $E^{k-1}$ by requiring it to satisfy condition B for all vectors tangent to the fiber, and requiring it to reduce to $\Gamma^{k}$ on vectors tangent to $E^{k-1}$. Next we define it on all of $E$ by condition $C$. We will denote this extension of $\Gamma^{k}$ by $\Gamma$. It is clearly a connection form on $E$. Connections arising this way are what Ehresmann calls Cartan connections (cf. Ehresmann [5]).

The connection defined by $\Gamma$ can also be thought of as a connection on the homogeneous space bundle $E / E^{k-1}$ with fiber $H / G^{k-1}$. By Theorem 5.1 this connection is flat if and only if the original $G$-structure is integrable. 
It is also possible to associate Cartan connections with $G$-structures which are uniformly $(k-1)$-flat. The construction requires, however, certain supplementary hypotheses about $G$. For example, it is enough to assume that if $Z^{k-1,2}$ is the space of cocycles in $C^{k-1,2}(g)$ then there is an invariant complement to $\partial C^{k, 1}$ in $Z^{k-1,2}$. Supposing this hypothesis satisfied, denote by $D$ a particular invariant complement to $\delta C^{k, 1}$ in $Z^{k-1,2}$. By Proposition 4.3, at each point of $E^{k-1}$ there exists a unique horizontal space, $H$, annihilating the $g+g^{(1)}+\cdots+g^{(k-2)}$ component of $\Gamma^{k-1}$ and such that $\Pi_{H}^{(k-2)}$ is in $D$. This horizontal space gives one a way of identifying the tangent space to $E^{k-1}$ at the point in question with the tangent space to $E_{0}^{k-1}$ at $i_{k-1}$. In other words, by choosing $D$, we get a $\left(V+g+\cdots+g^{(k-1)}\right)$ valued one-form on $E^{k-1}$. This form satisfies the properties $\mathrm{A}, \mathrm{B}, \mathrm{C}$; therefore just as above, it defines a Cartan connection on $E$.

As an example we consider the conformal group. By Proposition 6.2 it is of type 2, and by Proposition 6.3 every conformal structure is uniformly one-flat. Moreover, since the conformal group is reductive one can always find an invariant subspace, $D$, of $Z^{1,2}$ complementary to $\delta C^{2,1}(g)$. Therefore every conformal structure defines a Cartan connection. This connection turns out to be a spherical connection; the associated homogeneous space bundle is an $n$-sphere bundle. The existence of this connection was pointed out by Cartan in [3].

8. The tensors of structure computed in terms of classical invariants. Let $E \rightarrow M$ be a $G$-structure and let $\omega$ be its structure form. Let $\Lambda$ be an arbitrary linear connection on $E$. At every point of $E, \Lambda$ defines a horizontal subspace, $H$, of the tangent space to $E$. The $V$-valued two-form, $d \omega \circ H$, is called the torsion form of $\Lambda$. It defines an element $\left(\Pi_{H}^{-1)}\right.$ in our previous notation) of $V \circ V^{*} \wedge V^{*}$ called the torsion tensor of the connection at the point in question. Therefore:

Proposition 8.1. Let $Q$ be an arbitrary point of $E$. The lowest order structure tensor of $E \rightarrow M$ at $Q$ is the cohomology class in $H^{0,2}(g)$ of the torsion tensor of $\Lambda$ at $Q$.

If this structure tensor vanishes, there exist linear connections on $E$ without torsion. In fact one can prove a somewhat more general result:

Each element of $G$ is a homogeneous linear transformation of $V$; therefore it defines an element of $G^{k}$ for all $k$. In other words there is a canonical imbedding of $G$ in $G^{k}$. For each $k$, therefore, we can think of $G$ as a subgroup of $G^{k}$.

Suppose the lowest order structure tensor of $E \rightarrow M$ vanishes. By our main theorem $E \rightarrow M$ is uniformly one-flat; and, therefore, the prolongation $E^{1} \rightarrow M$ is defined. We will prove:

Proposition 8.2. There is a one-one correspondence between torsionless 
connections on $E$ and cross sections of $E^{1} \rightarrow E$ which commute with $G$. Specifically, if $\phi: E \rightarrow E^{1}$ is a cross section of $E^{1} \rightarrow E$ commuting with $G$, the connection form of the associated connection is $\phi^{*} \Omega^{0}$, where $\Omega^{0}$ is the $g$ component of the structure form of $E^{1}$.

Proof. It is clear that if $\phi$ is such a cross section then $\phi^{*} \Omega^{0}$ is a connection form on $E$. Moreover it satisfies

$$
d \omega+\left[\phi^{*} \Omega^{0}, \omega\right]=0,
$$

which, as Nomizu has shown (cf. [8]), is equivalent to torsion vanishing. To establish the correspondence in the other direction suppose that $\Lambda$ is a connection form on $E$ satisfying

$$
d \Lambda+[\Lambda, \omega]=0 \text {. }
$$

Let $Q$ be a point of $E$. By an argument used in establishing Theorem 4.1 we can prove that the mapping of the tangent space to $E$ at $Q$ onto the tangent space to $E_{0}$ at $i_{0}$ defined by $(\omega, \Lambda)$ is induced by a two-jet preserving structure to first order at $Q$. This defines the required lifting $\phi: E \rightarrow E^{1}$; and $\phi^{*} \Omega^{0}=\Lambda$ is a consequence of the definitions. Q.E.D.

Let $Z^{1,2}(g)$ be the cocycles of bidegree $(1,2)$ in the Spencer $\delta$-chain complex and let $\Pi$ be the projection of $Z^{1,2}(g)$ onto $H^{1,2}(g)$.

Proposition 8.3. If $\Lambda$ is a torsionless connection on $E$ and $R$ is its curvature tensor, $R$ takes its values in $Z^{1,2}(g)$ and the first order structure tensor of $E$ is $\Pi R$.

Proof. Let $\phi$ be the cross section of $E^{\prime} \rightarrow E$ associated with $\Lambda$. Let $Q$ be a point of $E$ and $H$ the horizontal space at $Q$ defined by the connection. By definition $\phi_{*} H$ is annihilated by $\Omega^{0}$; therefore the structure tensor at $Q$ is the cohomology class of the element in $g \otimes V^{*} \wedge V^{*}$ associated with $d \Omega^{0} \circ \phi_{*} H$. But by definition $\phi^{*}$ of this is $d \Lambda \circ H$, which is the curvature form of $\Lambda$ at $Q$. Q.E.D.

Let us now choose, for once and for all, a torsionless connection on $E$ with connection form $\Lambda$.

Consider the following system of differential equations on $E$.

$$
\begin{gathered}
d \Lambda+\frac{1}{2}[\Lambda, \Lambda]+\left[\omega, \Lambda^{1}\right]=0 \\
d \Lambda^{1}+\frac{1}{2}\left(\left[\Lambda, \Lambda^{1}\right]+\left[\Lambda^{1}, \Lambda\right]\right)+\left[\omega, \Lambda^{2}\right]=0 \\
\vdots \\
\vdots \\
d \Lambda^{k-2}+\frac{1}{2}\left(\left[\Lambda, \Lambda^{k-2}\right]+\left[\Lambda^{1}, \Lambda^{k-3}\right]+\cdots+\left[\Lambda^{k-2}, \Lambda\right]\right)+\left[\omega, \Lambda^{k-1}\right]=0 .
\end{gathered}
$$

The term $\Lambda^{l}$ is to be a one-form taking its values in $g^{(l)}$, and the brackets are defined according to the conventions of $\$ 1$. A solution to these equa- 
tions will be called admissible if the forms $\Lambda^{l}, l>0$, are horizontal on $E$ (that is, zero when restricted to the fibers) and transform as forms of type $g^{(n)}$ under the action of $G$. Let $\phi$ be the cross section of $E^{\prime} \rightarrow E$ associated with the torsionless connection.

We will prove:

Proposition 8.4. If (8.1) has an admissible solution, then $E \rightarrow M$ is uniformly $k$-flat. Moreover, there is a one-one correspondence between admissible solutions to the system (8.1) and cross sections of $E^{k} \rightarrow E$ which extend $\phi$ and commute with $G$. Explicitly, if $\psi$ is such a cross section, $\psi^{*} \Omega^{l}, l=1$, $\ldots, k-1$, is an admissible solution to (8.1), where $\Omega^{l}$ is the $g^{(l)}$ component of the structure form on $E^{k}$.

Proof (by induction). The first step in the induction has been demonstrated by Proposition 8.2. We will assume that the system of equations (8.1) has been solved up to order $k-2$, which implies by induction that $E \rightarrow M$ is uniformly $(k-1)$-flat and that there exists a cross section $\psi$ : $E \rightarrow E^{k-1}$ such that $\Lambda^{l}=\psi^{*} \Omega^{l}, l=1, \cdots, k-2$.

Let $\Lambda^{k-1}$ be a $g^{(k-1)}$-valued one-form on $E$ satisfying

$$
d \Lambda^{k-2}+\frac{1}{2}\left(\left[\Lambda, \Lambda^{k-2}\right]+\cdots+\left[\Lambda^{k-2}, \Lambda\right]\right)+\left[\omega, \Lambda^{k-1}\right] .
$$

Let $Q$ be a point of $E$ and $H$ the horizontal space determined by the connection at $Q$. We will define a $g^{(k-1)}$-valued one-form at $\psi(Q)$ by requiring it to be equal to $\Lambda^{k-1}$ on $\psi_{*} H$ and to take the appropriate values on the vertical vectors corresponding to $g^{(1)}+\cdots+g^{(k-1)}$. By definition

$$
d \Omega^{k-2}+\frac{1}{2}\left(\left[\Omega^{k-2}, \Omega^{0}\right]+\left[\Omega^{k-3}, \Omega^{1}\right]+\cdots+\left[\Omega^{0}, \Omega^{k-2}\right]\right)+\left[\omega, \Omega^{k-1}\right]=0
$$

at $\psi(Q)$, and

$$
\psi^{*} \Omega^{k-1}=\Lambda^{k-1} .
$$

Referring again to the argument used to prove Theorem 4.1, the mapping of the tangent space to $E^{k-1}$ at $\psi(Q)$ onto the tangent space to $E_{0}^{k-1}$ at $i_{k-1}$ defined by $\left(\omega, \Omega^{0}, \cdots, \Omega^{k-1}\right)$ is induced by a $(k+1)$-jet lifting $\psi(Q)$ and preserving structure to $k$ th order at $Q$. Thus $E \rightarrow M$ is uniformly $k$ flat and $\Lambda^{k-1}$ defines a cross section $\psi^{\prime}: E \rightarrow E^{k}$ extending $\psi: E \rightarrow E^{k-1}$. Q.E.D.

Corollary. Suppose $E \rightarrow M$ is uniformly k-flat. Its kth structure tensor can be written as a polynomial expression in the curvature tensor of $\Lambda$ and its first $k-1$ covariant derivatives.

Proof. We will indicate the first stage of the proof. If $E \rightarrow M$ is uniformly two-flat then the equation 


$$
d \Lambda+\frac{1}{2}[\Lambda, \Lambda]+\left[\omega, \Lambda^{1}\right]=0
$$

has an admissible solution. Let $Q$ be a point of $E$. The expression $d \Lambda^{1}$ $+\left[\Lambda, \Lambda^{1}\right]$ defines an element of $g^{(1)} \otimes V^{*} \wedge V^{*}$ at $Q$, and this is easily seen to be a cocycle in the cohomology class of the tensor of structure at $Q$. However $\Lambda^{1}$ is a horizontal form whose coefficients, in terms of $\omega$, are linear expressions in the coefficients of the curvature tensor; and $d \Lambda^{1}$ $+\left[\Lambda, \Lambda^{1}\right]$ is the covariant derivative of $\Lambda^{1}$ (cf. Nomizu [8]). Therefore, it is a horizontal form whose coefficients (in terms of $\omega$ ) are linear expressions in the first covariant derivatives of the curvature tensor.

In general the $k$ th tensor of structure is determined by an expression of the form:

$$
D \Lambda^{k-1}+\frac{1}{2}\left(\left[\Lambda^{1}, \Lambda^{k-2}\right]+\cdots+\left[\Lambda^{k-2}, \Lambda^{1}\right]\right),
$$

where $D$ is covariant differentiation, and the forms $\Lambda^{1}, \cdots, \Lambda^{k-1}$ are an admissible solution to (8.1). By induction the coefficients of these forms in terms of $\omega$ are polynomial expressions in $R$ and its first $k-2$ covariant derivatives. Therefore, the $k$ th structure tensor is an expression in $R$ and its first $k-1$ covariant derivatives. Q.E.D.

\section{BiBLOGRAPHY}

1. D. Bernard, Sur la géométrie différentielle des G-structures, Ann. Inst. Fourier (Grenoble) 10 (1960), 151-270.

2. E. Cartan, Les groupes de transformations continues, infinies, simples, Ann. Sci. Ecole Norm. Sup. 26 (1909), 93-161.

3. $\longrightarrow$, Les espaces a connexion conforme, Ann. Soc. Polon. Math. 2 (1923), 171-221.

4. C. Chevalley, Théorie des groups de Lie, Tome II, Groupes algébriques, Hermann, Paris, 1951.

5. C. Ehresmann, Les connexions infinitésimales dans un espace fibré differentiable, Colloque de topologie, Brussels, 1950, pp. 29-55, Masson, Paris, 1951.

6. V. W. Guillemin and Shlomo Stemberg, An algebraic model of transitive differential geometry, Bull. Amer. Math. Soc. 70 (1964), 16-47.

7. Y. Matsushima, Sur les algèbres de Lie linéaires semi-involutives, Colloque de topologie de Strasbourg, 1954-1955, Inst. de Math., Université de Strasbourg, 17 pp.

8. K. Nomizu, Lie groups and differential geometry, The Mathematical Society of Japan, Tokyo, 1956.

9. J. A. Shouten, Über die konforme Abbildung n-dimensional Mannigfaltigkeiten mit quadratischer Massbestimmung auf eine Mannigfaltigkeit mit euklidscher Massbestimmung, Math. Z. 11 (1921), 55-58.

10. I. M. Singer and S. Sternberg, On the infinite groups of Lie and Cartan, J. Analyse Math. (to appear).

11. D. C. Spencer, Deformation of structures on manifolds defined by transitive continuous pseudogroups, Ann. of Math. (2) 76 (1962), 306-445.

12. S. Sternberg, Lectures on differential geometry, Prentice-Hall, Englewood Cliffs, N. J., 1963.

13. H. Weyl, Reine infinitesimal Geometrie, Math. Z. 2 (1918), 384-411. 